\title{
Comparative effectiveness of minimally invasive versus traditional sternotomy mitral valve surgery in elderly patients
}

\author{
Alexander Iribarne, MD, MS, ${ }^{\text {a }}$ Rachel Easterwood, BA, ${ }^{a}$ Mark J. Russo, MD, MS, ${ }^{b}$ Edward Y. Chan, MD, ${ }^{a}$ \\ Craig R. Smith, MD, ${ }^{a}$ and Michael Argenziano, MD $^{\mathrm{a}}$
}

Objectives: This study assessed comparative effectiveness of minimally invasive versus traditional sternotomy mitral valve surgery in elderly patients.

\begin{abstract}
Methods: From January 1, 2000, to December 31, 2008, 1005 patients underwent isolated mitral valve surgery at our institution. Patients $\geq 75$-years-old were included in analysis (sternotomy, $\mathrm{n}=105$; minimally invasive, $\mathrm{n}=70$ ). Clinical outcomes included bypass and crossclamp time, length of hospitalization, morbidity, and mortality. To assess resource use, total hospital costs and discharge location were analyzed. Three standardized inpatient functional status outcomes were also assessed.
\end{abstract}

\begin{abstract}
Results: The minimally invasive approach was associated with a 9.2-minute longer crossclamp time $(P=.037)$ and a 25.2 -minute longer bypass time $(P<.001)$. Minimally invasive surgery was associated with a 3.1-day shorter hospitalization $(P=.033)$. There were no significant differences in rate of major postoperative complications $(P=.085)$ or long-term survival $(P=.60)$. Minimally invasive approach was associated with a $\$ 6721$ lower median cost of hospitalization $(P=.007)$ and more common discharge to home, routinely or with a health aide, rather than to rehabilitation $(P=.021)$. Minimally invasive patients achieved faster rates of independent ambulation $(P=.039)$ and independent sit-to-stand activity $(P=.003)$, although there were no differences in time to independent stair climbing $(P=.31)$.
\end{abstract}

Conclusions: Among elderly patients, minimally invasive mitral valve surgery is associated with slightly longer crossclamp and bypass times but with equivalent morbidity and mortality and shorter hospitalization, decreased resource use, and improved postoperative functional status. (J Thorac Cardiovasc Surg 2012;143:S86-90)

Since the first descriptions of minimally invasive mitral valve surgery (MIMVS) in the mid 1990s, there has been a growing body literature on the safety and efficacy of minimally invasive approaches for both mitral valve repair and replacement. ${ }^{1}$ Some of the reported benefits of a minimal access approach include decreased surgical trauma, improved cosmesis, decreased postoperative pain, and reduced duration of hospitalization. ${ }^{2}$

Despite improvements in surgical technique and cannulation methods, the potential advantages of MIMVS have often been tempered by concerns regarding the efficacy of

From the Division of Cardiothoracic Surgery, ${ }^{\text {a }}$ Department of Surgery, College of Physicians and Surgeons, Columbia University, New York, NY; and the Section of Cardiac and Thoracic Surgery, ${ }^{\mathrm{b}}$ University of Chicago Medical Center, Chicago, Ill.

Supported in part by National Institutes of Health Training Grant 5T32HL007854-13 (to A.I.).

Disclosures: Alexander Iribarne, Rachel Easterwood, Mark J. Russo, Edward Y. Chan, MD, Craig R. Smith, and Michael Argenziano have nothing to disclose with regard to commercial support.

Presented at The American Association for Thoracic Surgery Mitral Conclave, New York, New York, May 5-6, 2011.

Received for publication May 30, 2011; revisions received Sept 12, 2011; accepted for publication Oct 5, 2011.

Address for reprints: Michael Argenziano, MD, Columbia University Medical Center, Milstein Hospital Building, Room 7-435, 177 Fort Washington Ave, New York, NY 10032 (E-mail: ma66@ columbia.edu).

$0022-5223 / \$ 36.00$

Copyright (C) 2012 Published by Elsevier Inc. on behalf of The American Association for Thoracic Surgery

doi:10.1016/j.jtcvs.2011.10.090 mitral valve surgery performed under limited exposure, the learning curve associated with minimally invasive approaches, and the potential for prolonged operative times. Although no prospective studies have compared MIMVS to the traditional sternotomy approach, several retrospective analyses have shown that the potential benefits of MIMVS can be achieved without compromise to the efficacy of valve repair and without increased morbidity or mortality. ${ }^{3,4}$ One of the major limitations of such analyses, however, is that often significant differences in baseline risk exist between minimally invasive and sternotomy groups, and thus statistical adjustments are required to compare groups, which results in inherent bias. ${ }^{5}$ In most analyses, patients undergoing MIMVS are at lower risk, are younger, have a lower body mass index, and have fewer comorbidities.

As the popularity of MIMVS has grown, the technique has been expanded to patient subgroups previously considered at too high risk for a minimally invasive approach. Currently, there is a paucity of literature on minimally invasive cardiac surgery in the elderly population. The goal of this analysis was therefore to assess the comparative effectiveness of a minimally invasive versus a traditional sternotomy approach for mitral valve surgery in elderly patients.

\section{MATERIALS AND METHODS \\ Study Population}

After institutional review board approval was obtained, data on all isolated mitral valve operations performed at our institution from January 1, 


\section{Abbreviations and Acronyms \\ $\mathrm{CPB}=$ cardiopulmonary bypass \\ MIMVS $=$ minimally invasive mitral valve surgery}

2000, to December 31, 2008 ( $\mathrm{n}=1005)$ were retrospectively reviewed. Isolated mitral valve surgery was defined as any mitral valve repair or replacement performed in the absence of a major concomitant procedure, such as coronary artery bypass grafting or other valve surgery. Patients younger than 75 years and those undergoing reoperative surgery were excluded from the analysis for a net effective study cohort of 175 patients. A traditional median sternotomy approach was used in 105 patients $(60 \%)$, and a minimally invasive approach was used in 70 patients $(40 \%)$.

\section{Operative Technique}

Although MIMVS can be defined broadly according to the Society of Thoracic Surgeons database guidelines as any mitral valve repair or replacement performed through an incision other than a full median sternotomy, in our analysis all MIMVS cases were performed through a right minithoracotomy. Our operative approach for MIMVS performed through a minithoracotomy has been described previously elsewhere. ${ }^{6}$ Of note, aortic cannulation was most commonly performed in a central fashion, and venous drainage was most commonly achieved through a percutaneous femoral vein approach with single multistage venous cannulation. Central aortic cannulation was performed through the initial thoracotomy incision, and a transthoracic aortic crossclamp (Chitwood clamp; Scanlan International Inc, Minneapolis, Minn) was passed through a stab wound in the right axilla.

\section{Outcome Measures}

Major clinical outcome measures included cardiopulmonary bypass (CPB) time, crossclamp time, duration of hospitalization, major in-hospital complications, (intubation $>72$ hours, renal failure, sepsis, reoperation for bleeding, stroke, and gastrointestinal bleeding), and both short- and long-term survivals. To assess resource use, data were also collected on total hospital costs, direct costs, indirect costs, number of readmissions within 1 year of surgery, and discharge location. Hospital cost data were obtained from our institutional finance department decision support system (Eclipsys Transition System Incorporated, Atlanta, Ga), which provides audited clinical, financial, and operational data. The reported cost data represent actual resource costs to the hospital for services rendered, rather than billed charges. Finally, to assess the effect of incision type on functional status, data on time to independent sit-to-stand, time to independent ambulation, and time to independent stair climbing were gathered from standardized computer-entry inpatient physical therapy forms.

\section{Statistical Analysis}

Continuous variables are reported as mean $\pm \mathrm{SE}$ and median values and were compared with the Student $t$ test and Wilcoxon rank sum test, respectively. Categoric variables are reported as percentages and were compared with the $\chi^{2}$ test or Fisher's exact test as appropriate. Long-term survival, freedom from reoperation, and time to independent physical therapy milestones were assessed with the Kaplan-Meier method and compared with the log-rank test. Survival data were obtained from the Social Security Death Index and provided through January 1, 2010. All reported $P$ values are two-sided. All statistical analyses were performed with the Stata 11 statistical software package (StataCorp LP, College Station, Tex).

\section{RESULTS \\ Study Population}

Between January 1, 2000, and December 31, 2008, a total of 175 patients at least 75-years-old underwent isolated mitral valve surgery at our institution (sternotomy, $\mathrm{n}=$ $105,60 \%$; MIMVS, $\mathrm{n}=70,40 \%$ ). Baseline demographic characteristics are summarized in Table 1. Among 13 demographic variables, there were no significant differences in baseline risk between groups. There was no difference in the proportion of mitral repairs versus replacements between groups $(P=.753)$, and repair was more common than replacement in both sternotomy $(59.1 \%, \mathrm{n}=62)$ and MIMVS $(62.9 \%, \mathrm{n}=44)$ groups. Most patients in each group had mitral regurgitation rather than stenosis (sternotomy, $\mathrm{n}=92,87.6 \%$; MIMVS, $\mathrm{n}=59,84.3 \% ; P=.741$ ). Valve disease etiologies are summarized in Table 1 , and there was no significant difference between groups $(P=$ .345). Most patients had degenerative mitral valve disease (sternotomy, $\mathrm{n}=73,69.5 \%$; MIMVS, $\mathrm{n}=43,61.4 \%$ ); posterior leaflet prolapse was most common in both groups. Of note, among patients with degenerative disease, there was no significant difference in the repair rate between groups (sternotomy, $\mathrm{n}=43,58.9 \%$; MIMVS, $\mathrm{n}=23,53.5 \%$; $P=.70)$. There were no conversions to a median sternotomy approach in the MIMVS group.

\section{Clinical Outcomes}

With regard to intraoperative measures, crossclamp time was $9.2 \pm 2.4$ minutes longer (sternotomy, $75.2 \pm 2.4 \mathrm{~min}$ utes; MIMVS, $84.4 \pm 4.0$ minutes; $P=.037$ ), and CPB time was $25.2 \pm 5.7$ minutes longer (sternotomy, $110.4 \pm$ 3.0 minutes; MIMVS, $135.7 \pm 5.3$ minutes; $P<.001$ ) in the MIMVS group. The MIMVS group had a $3.1 \pm 1.4$ days shorter mean duration of hospitalization (sternotomy, $11.7 \pm 1.1$ days; MIMVS, $8.7 \pm 0.72$ days; $P=.033$ ). The median duration of hospitalization was 1 day shorter in the MIMVS group (sternotomy, 9 days; MIMVS, 8 days; $P=.038$ ). There was no significant difference in the overall rate of major postoperative complications between groups $(P=.085$; Table 1$)$. There was no difference between groups in survivals at 30 days $(P=.188)$ and at 1 year $(P=.902$; Table 1$)$. Also, there was no difference in Kaplan-Meier survival $(P=.60)$. The mean duration of survival follow-up was $3.8 \pm 2.4$ years.

\section{Resource Use}

Patients undergoing MIMVS had a $\$ 14,391 \pm \$ 5782$ lower mean cost of hospitalization (sternotomy, $\$ 60,289$ $\pm \$ 4843$; MIMVS, $\$ 45,897 \pm \$ 2586 ; P=.014)$. Patients undergoing MIMVS also had a significantly lower median cost of hospitalization by $\$ 6721$ (sternotomy, $\$ 43,790$; MIMVS, $\$ 37,069 ; P=.007)$. The mean difference in direct cost between groups was $\$ 9182 \pm \$ 3804$ (sternotomy, $\$ 36,387 \pm \$ 3194 ;$ MIMVS, $\$ 27,205 \pm \$ 1654 ; P=.017$ ), and the mean difference in indirect cost was $\$ 5372 \pm$ \$2544 (sternotomy, $\$ 24,065 \pm \$ 2027$; MIMVS, $\$ 18,692$ $\pm \$ 1378 ; P=.036)$. There was a significant association between incision type and discharge location $(P=.021)$, with 
TABLE 1. Baseline characteristics and major postoperative outcomes

\begin{tabular}{|c|c|c|c|}
\hline & $\begin{array}{c}\text { Sternotomy } \\
(\mathbf{n}=\mathbf{1 0 5})\end{array}$ & $\begin{array}{c}\text { Minimally } \\
\text { invasive } \\
(\mathbf{n}=\mathbf{7 0}) \\
\end{array}$ & $\begin{array}{c}P \\
\text { value }\end{array}$ \\
\hline \multicolumn{4}{|l|}{ Baseline variables } \\
\hline Age $(y$, mean $\pm S E)$ & $79.4 \pm 3.9$ & $78.6 \pm 3.3$ & .123 \\
\hline Male sex (no.) & $70(66.7 \%)$ & $43(61.4 \%)$ & .478 \\
\hline $\begin{array}{l}\text { Body mass index } \\
\qquad\left(\mathrm{kg} / \mathrm{m}^{2}, \text { mean } \pm \mathrm{SE}\right)\end{array}$ & $24.5 \pm 0.4$ & $24.0 \pm 0.5$ & .451 \\
\hline $\begin{array}{l}\text { Ejection fraction } \\
\quad(\%, \text { mean } \pm \mathrm{SE})\end{array}$ & $50.7 \% \pm 1.2 \%$ & $51.9 \% \pm 1.2 \%$ & .511 \\
\hline $\begin{array}{l}\text { Serum creatinine } \\
\quad(\mathrm{mg} / \mathrm{dL}, \text { mean } \pm \mathrm{SE})\end{array}$ & $1.1 \pm 0.04$ & $1.0 \pm 0.04$ & .319 \\
\hline COPD (no.) & $13(12.4 \%)$ & $4(5.7 \%)$ & .145 \\
\hline Diabetes (no.) & $7(6.7 \%)$ & $5(7.1 \%)$ & .903 \\
\hline Current CHF (no.) & $39(37.1 \%)$ & $18(25.7 \%)$ & .114 \\
\hline Renal failure (no.) & $2(1.9 \%)$ & $0(0 \%)$ & .245 \\
\hline $\begin{array}{r}\text { Cerebrovascular } \\
\text { accident (no.) }\end{array}$ & $9(8.6 \%)$ & $3(4.3 \%)$ & .216 \\
\hline $\begin{array}{l}\text { Immune system } \\
\text { deficiency (no.) }\end{array}$ & $7(6.7 \%)$ & $4(5.7 \%)$ & .799 \\
\hline $\begin{array}{l}\text { Peripheral vascular } \\
\text { disease (no.) }\end{array}$ & $2(1.9 \%)$ & $2(2.9 \%)$ & .680 \\
\hline Smoker (no.) & $20(19.1 \%)$ & $22(31.4 \%)$ & .060 \\
\hline Valve disease etiology (no.) & & & .345 \\
\hline Degenerative & $73(69.5 \%)$ & $43(61.4 \%)$ & \\
\hline Dilated cardiomyopathy & $6(5.7 \%)$ & $3(4.3 \%)$ & \\
\hline Endocarditis & $4(3.8 \%)$ & $3(4.3 \%)$ & \\
\hline Ischemic & $2(1.9 \%)$ & $6(8.6 \%)$ & \\
\hline Rheumatic & $4(3.8 \%)$ & $5(7.1 \%)$ & \\
\hline Calcific & $16(15.2 \%)$ & $10(14.3 \%)$ & \\
\hline \multicolumn{4}{|l|}{ Outcomes } \\
\hline $\begin{array}{l}\text { Crossclamp time } \\
\quad(\text { min, mean } \pm \mathrm{SE})\end{array}$ & $75.2 \pm 2.4$ & $84.4 \pm 4.0$ & .0374 \\
\hline $\begin{array}{l}\text { Bypass time (min, } \\
\text { mean } \pm \mathrm{SE})\end{array}$ & $110.4 \pm 3.0$ & $135.7 \pm 5.3$ & $<.001$ \\
\hline $\begin{array}{l}\text { Hospitalization } \\
\qquad(\mathrm{d}, \text { mean } \pm \mathrm{SE})\end{array}$ & $11.7 \pm 1.1$ & $8.7 \pm 0.72$ & .033 \\
\hline $\begin{array}{l}\text { Total hospital costs (US\$, } \\
\text { mean } \pm \text { SE) }\end{array}$ & $\$ 60,289 \pm \$ 4843$ & $\$ 45,897 \pm \$ 2586$ & .014 \\
\hline \multicolumn{4}{|l|}{ Morbidity (no.) } \\
\hline Intubation $>72 \mathrm{~h}$ & $14(13.3 \%)$ & $7(10 \%)$ & .506 \\
\hline Renal failure & $6(5.7 \%)$ & $1(1.4 \%)$ & .156 \\
\hline Sepsis & $1(0.95 \%)$ & $2(2.9 \%)$ & .342 \\
\hline $\begin{array}{l}\text { Reoperation for } \\
\text { bleeding }\end{array}$ & $5(4.8 \%)$ & $0(0 \%)$ & .064 \\
\hline Stroke & $7(6.7 \%)$ & $2(2.9 \%)$ & .264 \\
\hline $\begin{array}{l}\text { Gastrointestinal } \\
\text { bleeding }\end{array}$ & $1(0.95 \%)$ & $2(2.9 \%)$ & .342 \\
\hline \multicolumn{4}{|l|}{ Mortality (no.) } \\
\hline $30-d$ & $3(2.8 \%)$ & $5(7.1 \%)$ & .188 \\
\hline $1-y$ & $11(10.6 \%)$ & $7(10 \%)$ & .902 \\
\hline
\end{tabular}

most patients undergoing MIMVS discharged home routinely or with a home health aide (sternotomy, 58.2\%; MIMVS, $78.1 \%$ ) and significantly more of those undergoing sternotomy discharged to a rehabilitation facility (sternotomy, $41.7 \%$; MIMVS, $21.8 \%$ ). There was no difference in the rate of readmissions at 1 year between groups $(P=.542)$.

\section{Functional Status}

There was no significant difference in time to first physical therapy visit between the MIMVS and sternotomy groups $(P=.14)$. A time-to-event comparison of the 3 major functional status outcomes is summarized in Figure 1. Patients in the MIMVS group achieved faster rates of independent ambulation $(P=.039)$ and independent sit-to-stand activity $(P=.003)$; however, there was no difference in time to independent stair climbing $(P=.31)$.

\section{DISCUSSION}

Elderly persons are among the fastest growing segments of the population in the United States, and recent projections indicate that during the next 50 years the number of individuals older than 75 years will quadruple. ${ }^{7}$ Data from the Framingham Heart Study have demonstrated an increase in prevalence of moderate mitral regurgitation from $2.4 \%$ among patients between the ages of 60 and 69 years to $11.2 \%$ among patients between the ages of 70 and 83 years. ${ }^{8}$ Previous studies have demonstrated that cardiac surgery can be performed on elderly patients with higher, albeit improving, perioperative morbidity and mortality; however, the increase in prevalence of this population demands innovative approaches that not only address perioperative risk but also postoperative resource use and functional status. ${ }^{9}$

MIMVS has grown in popularity during the past decade, and with improvements in technology and surgical technique, minimally invasive surgery has been expanded to patient subgroups previously not considered candidates for this approach. ${ }^{10}$ There is a paucity of literature on outcomes of MIMVS in the elderly population. In this analysis, we have demonstrated that MIMVS can be performed safely in patients at least 75 years old. Although the minimally invasive approach was associated with slightly longer CPB and crossclamp times than was the conventional sternotomy approach, there were no significant differences in postoperative morbidity and mortality. Importantly, patients undergoing MIMVS had approximate 3 days shorter mean and 1 day shorter median durations of hospitalization, a finding that has important implications for resource use.

There were significant reductions in both mean and median costs of hospitalization associated with the minimally invasive approach, a finding that correlates with the observed difference in duration of hospitalization found between the groups. Similar differences were also observed in mean direct and indirect costs. Because of the rightskewed distribution of costs, the median difference in total hospital costs of $\$ 6721$ rather than the mean difference most likely represents the true cost savings. Most of the patients 

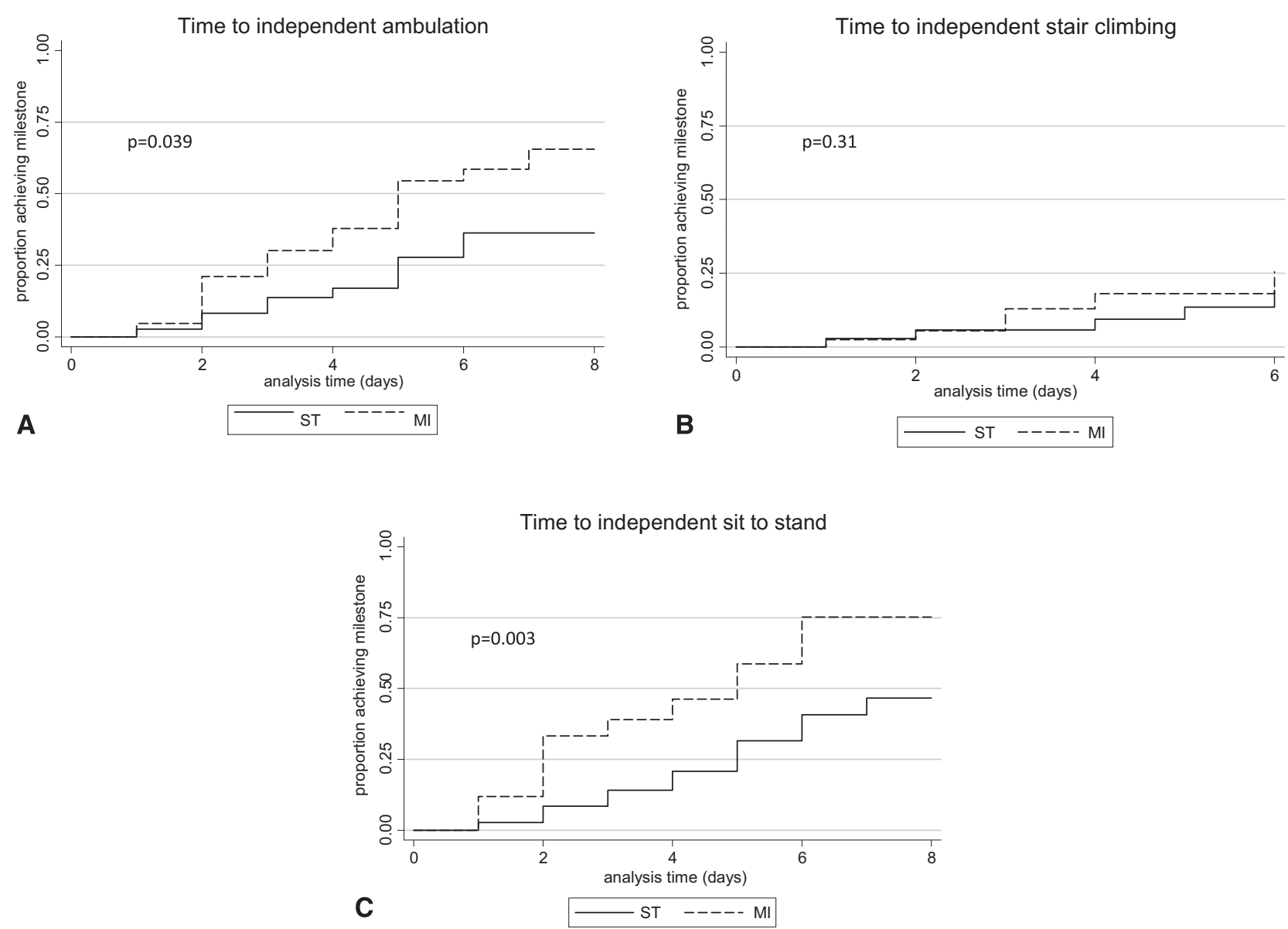

FIGURE 1. Kaplan-Meier estimates stratified by minimally invasive ( $M I)$ versus sternotomy (ST) approach. A, Time to independent ambulation; B, time to independent stair climbing; $\mathrm{C}$, time to independent sit-to-stand activity.

undergoing MIMVS were also discharged home without services or with a home health aide, as opposed to being discharged to a rehabilitation center, demonstrating the potential for significant future cost savings associated with the minimally invasive approach. Moreover, there were no differences in readmission rates at 1 year between groups. Of note, patients did not undergo any specific preoperative imaging studies to define eligibility for MIMVS, demonstrating that such imaging studies, which increase resource use, may not be routinely necessary for MIMVS.

Decisions for discharge location are most often based on a patient's postoperative functional status. At our institution, all patients receive inpatient physical therapy after surgery, and progress is recorded in standardized computer-entry forms. Patients undergoing MIMVS had faster rates for both time to independent ambulation and time to independent sit-to-stand activity. The observed shorter duration of hospitalization in that group thus may be due in part to faster inpatient physical rehabilitation, which translated into earlier discharge home. To date, no studies have assessed differences in postoperative functional status by incision type; however, our findings highlight a potential etiology for both the observed reduction in duration of hospitalization and the improved discharge location.

\section{Limitations}

There are several limitations to our analysis. First, our study is retrospective and subject to multiple potential biases. Although there was no difference in 13 baseline variables, other unrecorded differences may exist between groups, and selection bias cannot be completely eliminated. At our institution, the decision to pursue a minimally invasive approach involves an overall assessment of the feasibility and safety of such an approach according to the patient's preoperative risk. MIMVS is particularly challenging, for example, in treating patients with severe chronic obstructive pulmonary disease, elevated body mass index, and previous cardiac surgery. Our analysis was limited to isolated mitral valve disease, and reoperative cases were excluded. Also, although there were no significant differences in baseline risk, the relative proportion of patients with chronic obstructive pulmonary disease in the sternotomy group was higher.

Second, although data on postoperative functional status were standardized on computer order-entry forms and all 
cardiothoracic patients were evaluated by a physical therapist with specialization in thoracic physical therapy, there may have been variability in therapy provided. Such variability, however, would unlikely be biased toward either incision type versus the other.

Third, although it is our institutional practice to perform MIMVS through an incision that is generally between 6 and $8 \mathrm{~cm}$, in some instances it is possible that the thoracotomy incision may have been lengthened to improve exposure. In addition, although a minithoracotomy approach was used in this study, it should be noted that various less invasive incision types, such as a limited sternotomy, are used in practice and may potentially achieve similar results because of decreased surgical trauma.

Fourth, although there was no significant difference in the rate of repairs between groups, data on specific repair techniques were not available. These techniques may have influenced operative times.

Finally, the results do not take into account the learning curve associated with a minimally invasive approach and the fact that the observed advantages in resource use and functional status may not be achieved early in the learning process. Importantly, most of the minimally invasive cases were performed in the latter half of the study period, demonstrating that such an approach may not be readily applicable to higher-risk elderly patients early in the learning curve.

\section{CONCLUSIONS}

Although the study results must be interpreted in the context of the limitations cited here, we have demonstrated that MIMVS is not inferior to the traditional sternotomy approach with regard to morbidity and mortality, although it is associated with slightly increased crossclamp and CPB times. These increases in operative time are balanced, however, by the significant reduction in resource use and improvements in discharge disposition and inpatient function status achieved with a minimally invasive approach. In light of the growing elderly population, MIMVS represents an effective surgical approach for management of mitral valve disease with the potential for significant reductions in resource use.

\section{References}

1. Modi P, Hassan A, Chitwood WR Jr. Minimally invasive mitral valve surgery: a systematic review and meta-analysis. Eur J Cardiothorac Surg. 2008;34: 943-52.

2. McClure RS, Cohn LH, Wiegerinck E, Couper GS, Aranki SF, Bolman RM 3rd, et al. Early and late outcomes in minimally invasive mitral valve repair: an eleven-year experience in 707 patients. J Thorac Cardiovasc Surg. 2009;137: $70-5$.

3. Gammie JS, Bartlett ST, Griffith BP. Small-incision mitral valve repair: safe, durable, and approaching perfection. Ann Surg. 2009;250:409-15.

4. Greelish JP, Cohn LH, Leacche M, Mitchell M, Karavas A, Fox J, et al. Minimally invasive mitral valve repair suggests earlier operations for mitral valve disease. J Thorac Cardiovasc Surg. 2003;126:365-73.

5. Svensson LG, Atik FA, Cosgrove DM, Blackstone EH, Rajeswaran J, Krishnaswamy G, et al. Minimally invasive versus conventional mitral valve surgery: a propensity-matched comparison. J Thorac Cardiovasc Surg. 2010;139. 926-32.e1-2.

6. Iribarne A, Russo MJ, Easterwood R, Hong KN, Yang J, Cheema FH, et al. Minimally invasive versus sternotomy approach for mitral valve surgery: a propensity analysis. Ann Thorac Surg. 2010;90:1471-8.

7. Spencer G. U.S. Bureau of the Census: projections of the population of the United States, by age, sex and race: 1998-2080. Curr Popul Rep Popul Estim Proj. 1989;1018.

8. Singh JP, Evans JC, Levy D, Larson MG, Freed LA, Fuller DL, et al. Prevalence and clinical determinants of mitral, tricuspid, and aortic regurgitation (The Framingham Heart Study). Am J Cardiol. 1999;83:897-902.

9. Forman DE, Rich MW, Alexander KP, Zieman S, Maurer MS, Najjar SS, et al. Cardiac care for older adults. Time for a new paradigm. J Am Coll Cardiol. 2011;57:1801-10.

10. Schmitto JD, Mokashi SA, Cohn LH. Minimally-invasive valve surgery. $J$ Am Coll Cardiol. 2010;56:455-62. 